\title{
Method for the Calculation of DPA in the Reactor Pressure Vessel of Atucha II
}

\author{
J. A. Mascitti and M. Madariaga \\ Gerencia de Licenciamiento y Control de Reactores Nucleares, Autoridad Regulatoria Nuclear, Avenida Del Libertador 8250, \\ Ciudad Autónoma de Buenos Aires 1429, Argentina
}

Correspondence should be addressed to J. A. Mascitti, jmascitti@arn.gob.ar

Received 26 July 2010; Accepted 30 November 2010

Academic Editor: Alejandro Clausse

Copyright ( 2011 J. A. Mascitti and M. Madariaga. This is an open access article distributed under the Creative Commons Attribution License, which permits unrestricted use, distribution, and reproduction in any medium, provided the original work is properly cited.

\begin{abstract}
One of the limiting factors of the life of a nuclear power plant (NPP) is the state of the reactor pressure vessel (RPV). Embrittlement is the most important effect affecting RPV aging. The irradiation with neutrons, especially fast neutrons, is the primary cause of this embrittlement. NPP safe operation requires to ensure RPV integrity over its lifetime, threatened by the neutron radiation-induced embrittlement. In this paper, we identify the areas where the RPV neutron radiation is maximum and perform calculations of the displacement-per-atom (DPA) rate in those areas using the MCNP5 code. It was determined that the maximum DPA rate in the RPV wall with fresh fuel element (FE) is 3.76(3) $\times 10^{-12} \mathrm{~s}^{-1}$, it takes place in front of FEs BA42 and BL43, and it is symmetrical about the central channel, LG04, and LH03.
\end{abstract}

\section{Introduction}

Structural materials in a nuclear reactor are damaged by radiation from fission reactions. Irradiation displaces an atom from its site, leaving a vacant site behind (a vacancy), and the displaced atom eventually comes to rest in a location among lattice sites, becoming an interstitial atom. The interstitial vacancy pair is of main importance for radiation effects in solids and is known as a Frenkel pair (FP). The presence of the Frenkel pair and other consequences of irradiation damage determine the physical effects, and with the application of stress, the mechanical effects of irradiation. The radiation damage event is finished when the displaced atom (also known as the primary knock-on atom, PKA) comes to rest in the lattice as an interstitial [1] (Figure 1).

Damage in a material creates defects; defects can diffuse and create larger defects, leading to embrittlement of structural materials.

The safe operation of nuclear power plants requires ensuring, over the lifetime of the facility, the integrity of RPV threatened by the embrittlement induced by neutron radiation.
In this paper, we identify the areas where the neutron radiation flux in the RPV is maximum and perform calculations of the rate of DPA, the neutron flux, and neutron spectra in those areas with MCNP5 [2], a Monte Carlo code that can calculate neutron flux or fluence, and we compare the results with the values presented by NA-SA (the utility) in Section 4.3 of the Final Safety Analysis Report (FSAR) of the Atucha II NPP.

\section{Atucha II Model in MCNP}

Atucha II has been modeled so as to maximize the advantages provided by the Monte Carlo codes, with an accurate representation of geometry and materials.

2.1. Characteristics of the Model. It is a three-dimensional representation of Atucha II as shown in Figure 2, which shows two axonometric views of the reactor.

In the first graph, we can see the fuel channels, and in the second one fuel channels are not represented, so that the moderator tank, the guide tubes of control rods, and detector 


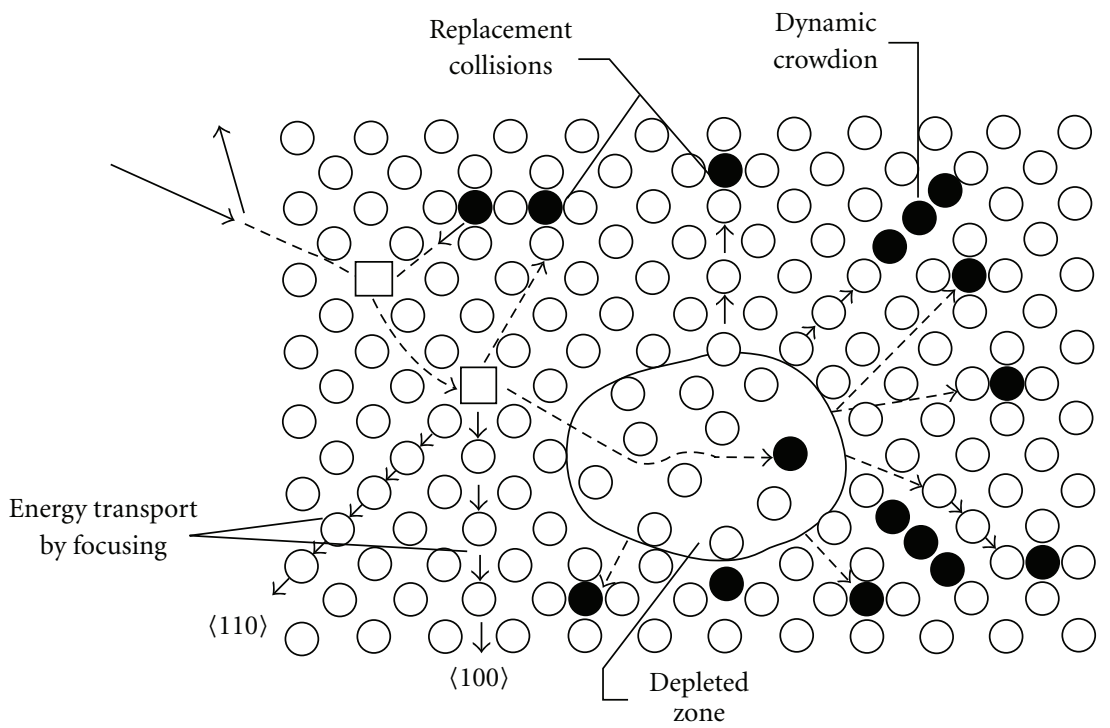

Figure 1: Schematic of neutron damage [1].

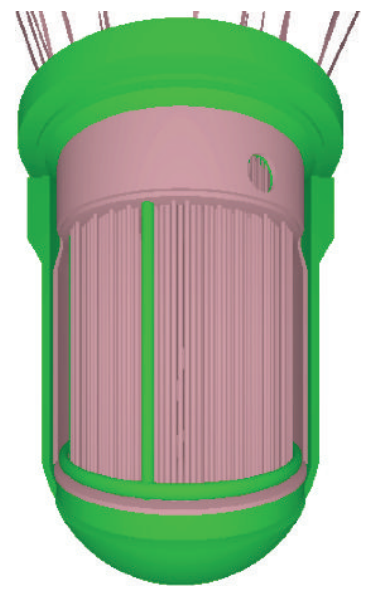

(a)

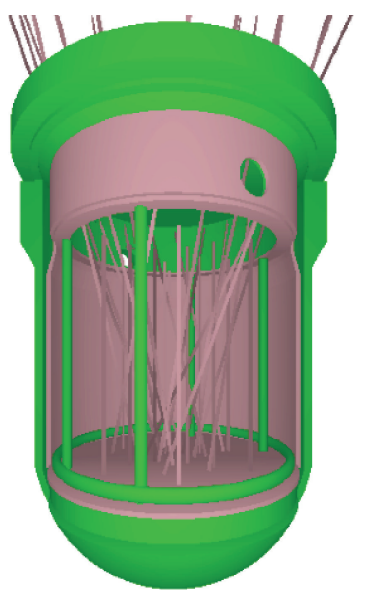

(b)
FIgURE 2: Axonometric view of the internal of the reactor, with and without fuel channels.

tubes may be appreciated. They were obtained from the MCNP5 input file with the graphical user interface visual editor that comes with the MCNP5 package software. A more detailed description of the elements included in the model can be seen in [3].

As for the state of the core for this first calculation, it was taken in a clean idealized state defined by fresh fuel, at hot full power without Xe and Sm, and with control rods out.

\subsection{Maximum Neutron Flux in the Inner Surface of the RPV}

Wall. Figure 3 shows the total neutron flux on the inner surface of the RPV wall. Total neutron flux is plotted in arbitrary units, according to Theta and $Z$ coordinates. The mid plane of the core is at $Z=150 \mathrm{~cm}$.

Transport to the RPV wall was made from a criticality calculation using increasing importance in the radial direction

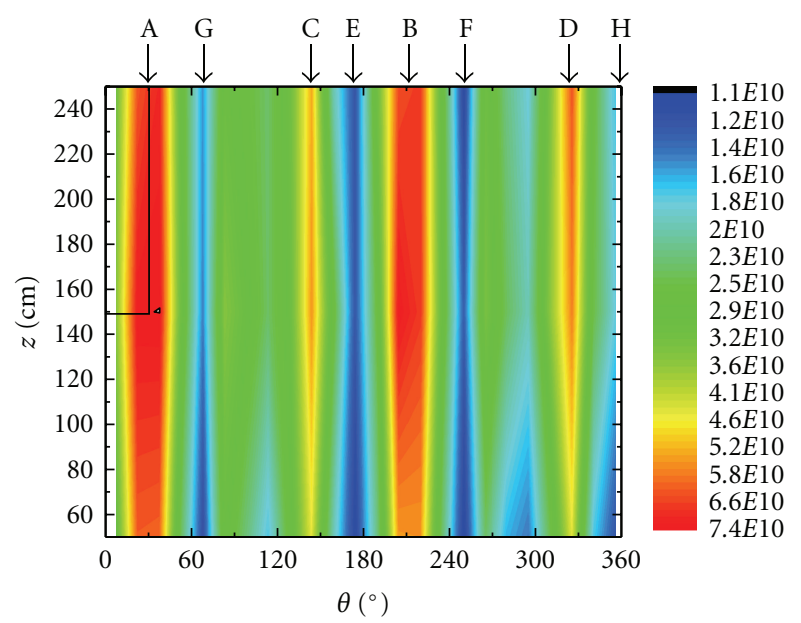

FIGURE 3: Total neutron flux on the inner surface of RPV (a.u.).

outside the fuel. Due to hardware limitations and although relatively extensive sections of wall were considered, it was not possible to obtain dispersions smaller than $10 \%$ for all the calculation points with the complete model as we would have liked to. This is a rough calculation that shows us zones with relatives maximum and minimum.

The graph shows the presence of two main peak areas A $\left(\theta=30^{\circ}\right)$ and $\mathrm{B}\left(\theta=210^{\circ}\right)$ and two secondary maxima $\mathrm{C}$ $\left(\theta=144^{\circ}\right)$ and $\mathrm{D}\left(\theta=328^{\circ}\right)$. It is also possible to observe the presence of minimum neutron flux in areas $\mathrm{E}\left(\theta=170^{\circ}\right)$, $\mathrm{F}\left(\theta=248^{\circ}\right), \mathrm{G}\left(\theta=70^{\circ}\right)$, and $\mathrm{H}\left(\theta=350^{\circ}\right)$. In Figure 3, we added a window denoting the region of interest at the last part of the work.

Figure 4 shows a reactor cross-section at the same positions as in the previous figure, showing that the principal maxima are in opposite positions in the area where there are more FEs (4) near the wall of the RPV. The same occurs for 


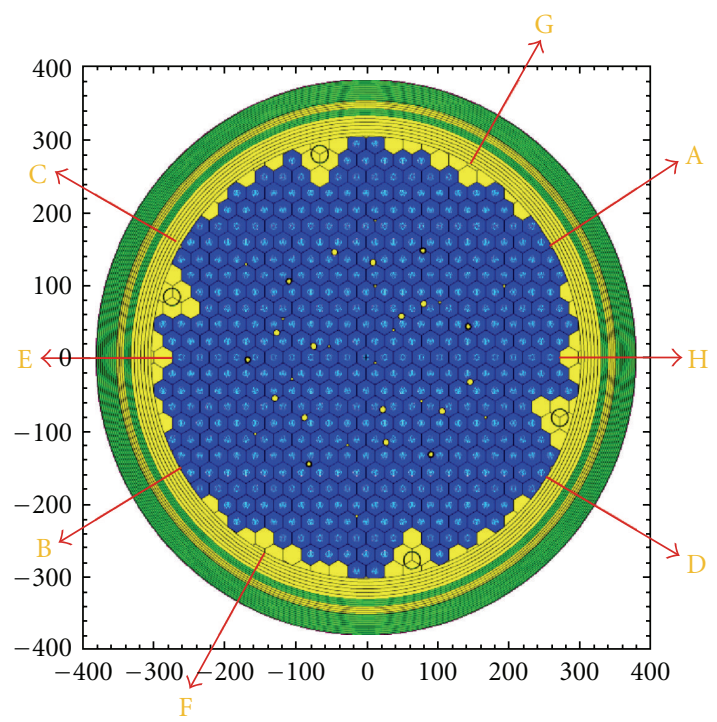

FIgURE 4: $x-y$ view of the reactor for $Z=0.0 \mathrm{~cm}$.

the secondary maxima but with $3 \mathrm{FE}$. Because of symmetry, each maximum and minimum has its equivalent separated by $180^{\circ}$.

In zone A, fuels 446 (AK44), 434 (BL43), 440 (BA42), and 450 (BB41) are located. In area B, fuels are 448 (LK02), 437 (LH03), 443 (LG04), and 451 (LF05). Brackets indicate the corresponding FEs with their name used in CNA II.

From these observations, we can consider the calculation of neutron damage focusing our attention on the first sixth of the reactor where the area $\mathrm{A}$ is located.

\section{Simplified Model}

A usual method of variance reduction in MCNP that decrease computing time is the truncation of the geometry.

3.1. First Sixth of the Reactor. Following the work [4] where it is shown that the contribution to neutron flux in the RPV wall is mainly due to the first two rows of fuel elements closest to the vessel wall, if the contribution of internal rows is negligible, only the three outer rows were modeled here as shown in Figure 5.

The results can be considered correct at the areas not too close to the edges $\left(\theta=0^{\circ}\right.$ and $\left.\theta=60^{\circ}\right)$, which is acceptable since we know that the maximum neutron flux is found around $\theta=30^{\circ}$.

\subsection{Truncation of Geometry Using Symmetry. From Figure 5,} we can see that the geometry is symmetrical with respect to the $\theta=30^{\circ}$ plane, which together with the symmetry plane at $z=0 \mathrm{~cm}$ and appropriate boundary conditions, enables us to keep truncating the geometry to $1 / 24$ the volume of the reactor as shown in Figure 6.

On surfaces $\theta=30^{\circ}$ and $z=0 \mathrm{~cm}$ reflective boundary conditions were imposed; at $\theta=0^{\circ}$, on the inner cylindrical segment, white boundary conditions were used, and other

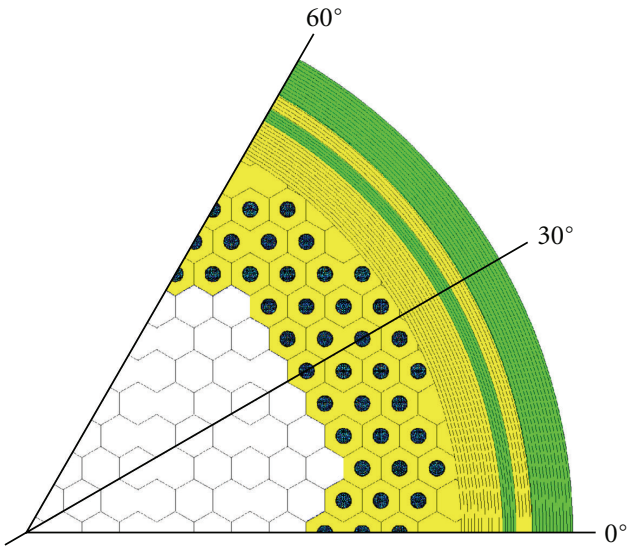

FIgURe 5: Axial view of the sixth reactor with three rows of $\mathrm{FE}$ closest to the RPV (sector $0^{\circ}$ to $60^{\circ}$ ).

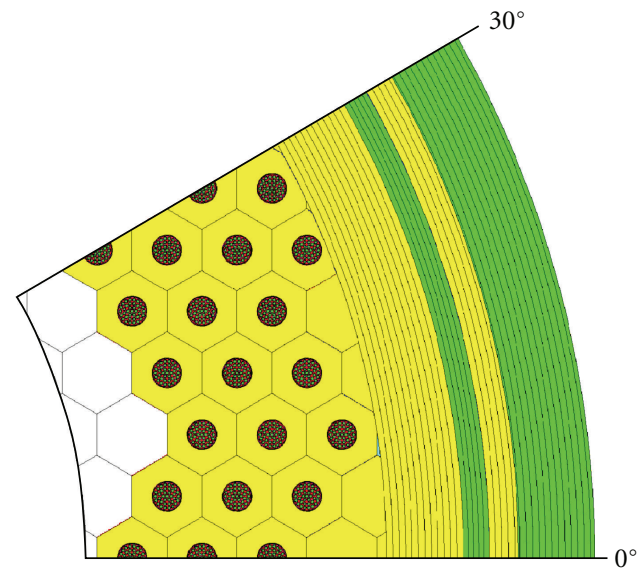

FIgURE 6: Scheme $1 / 24$ of the reactor.

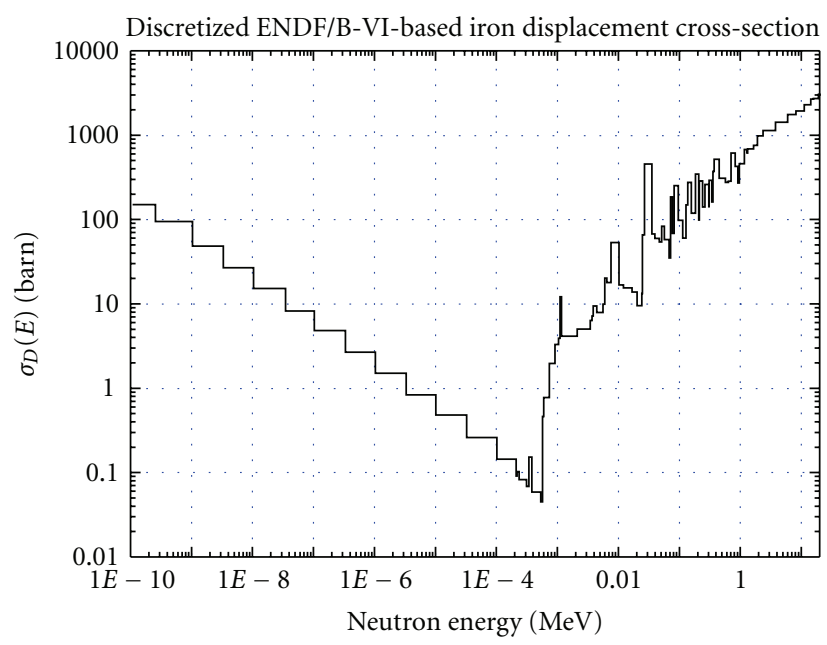

FIGURE 7: Discretized displacement cross-section for stainless steel based on a Lindhard model and ENDF/B scattering cross-sections. 


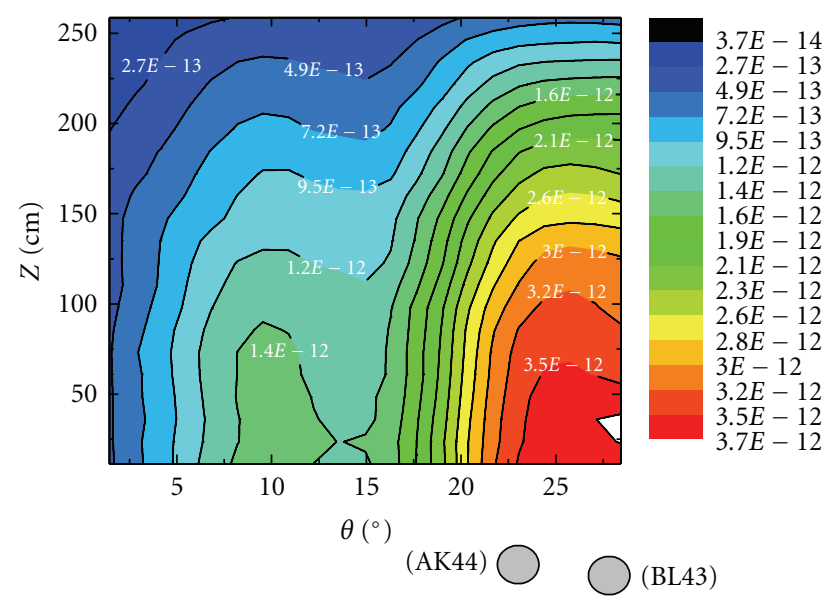

FIGURE 8: Rate of DPA in terms of $Z$ and Theta on the inner surface of the RPV $(369.5 \mathrm{~cm})$.

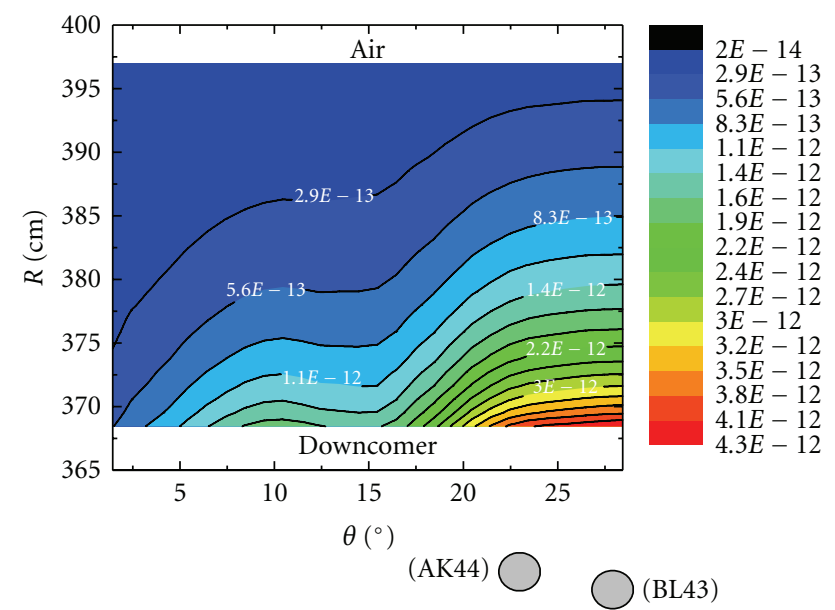

Figure 9: Rate of DPA on the RPV in terms of $R$ and Theta for $Z=33.75 \mathrm{~cm}$ with maximum DPA.

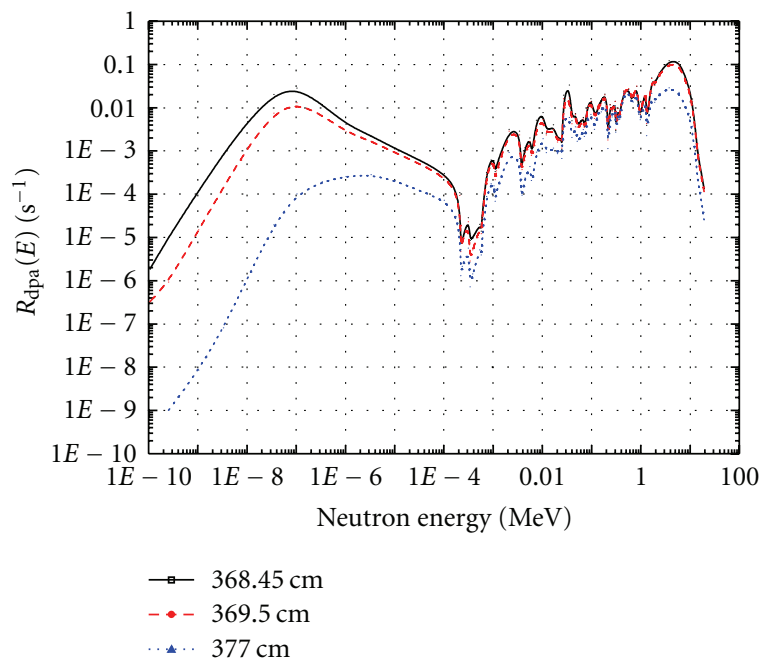

Figure 10: DPA rate versus neutron energy on the inner surface of the cladding, on the inner surface of the RPV, and $1 / 4 \cdot T$ for $\theta=28.44^{\circ}$ and $Z=33.75 \mathrm{~cm}$.

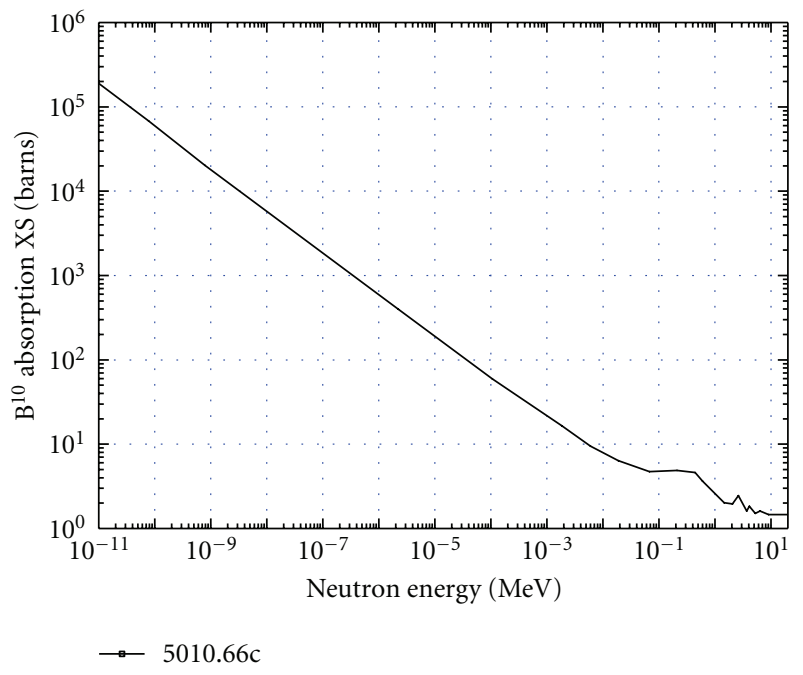

FIGURE 11: $\mathrm{B}^{10}$ total neutron disappearance cross-section (MT 101) from ENDF/B-VI library used by MCNP5.

areas lead to zero importance regions (equivalent to imposing black border conditions).

\section{Displacement Per Atom (DPA)}

A standard parameter in the determination of radiation damage in materials is the displacement per atom (DPA), an integral magnitude that includes information about the material response (displaced atoms) and the neutron fluence (magnitude and spectrum) to which the material was exposed. DPA is not a measure of initially created lattice defects in the material but a measure of the harming energy deposited by neutrons in terms of the number of atoms permanently displaced from their position to a stable interstitial position. DPA is the magnitude usually used to correlate damage on materials irradiated under different neutron environments.

4.1. Rate of DPA. The DPA rate is a derived quantity, which can be obtained dividing $R$, the number of displacements per unit volume, and time by the atomic density $N$ of the material (steel in this case),

$$
R_{\mathrm{DPA}}=\frac{R}{N}=\int_{E_{m}}^{E_{M}} \sigma_{D}\left(E_{i}\right) \Phi\left(E_{i}\right) d E_{i}
$$

where $N$ is the atom number density, $E_{M}$ is the maximum energy of the incoming particle, is the $E_{m}$ minimum energy of the incoming particle, $\Phi\left(E_{i}\right)$ is the energy-dependent particle flux per unit energy, and $\sigma_{D}\left(E_{i}\right)$ is the energydependent displacement cross-section.

With MCNP5 the neutron flux is calculated as a function of energy on the RPV, wall and it is combined with the DPA cross-section [5] in the pressure vessel steel (Figure 7). 
TABLE 1: Flux and DPA rate spectrum for $R=369.5 \mathrm{~cm}$ (RPV inner surface) and $R=377.0 \mathrm{~cm}(1 / 4 \cdot T)$.

\begin{tabular}{|c|c|c|c|c|c|c|c|c|c|c|}
\hline & & & \multicolumn{4}{|c|}{ Flux spectrum } & \multicolumn{4}{|c|}{ DPA rate spectrum } \\
\hline \multirow{2}{*}{\multicolumn{3}{|c|}{$\begin{array}{c}\text { Energy group } \\
\mathrm{MeV}\end{array}$}} & \multicolumn{2}{|c|}{ RPV inner surf. } & \multicolumn{2}{|c|}{$1 / 4 \cdot T$} & \multicolumn{2}{|c|}{ RPV inner surf. } & \multicolumn{2}{|c|}{$1 / 4 \cdot T$} \\
\hline & & & \multirow{2}{*}{$\frac{\mathrm{n} / \mathrm{cm}^{2} / \mathrm{s}}{2.12(1) E+10}$} & \multirow{2}{*}{$\frac{\% f}{26 \%}$} & \multirow{2}{*}{$\frac{\mathrm{n} / \mathrm{cm}^{2} / \mathrm{s}}{3.82(1) E+08}$} & \multirow{2}{*}{$\frac{\% f}{2 \%}$} & \multirow{2}{*}{$\frac{\mathrm{s}^{-1}}{1.50(1) E-13}$} & \multirow{2}{*}{$\begin{array}{c}\% \mathrm{f} \\
4 \%\end{array}$} & \multirow{2}{*}{$\frac{s^{-1}}{6(1) E-15}$} & \multirow{2}{*}{$\frac{\% f}{0 \%}$} \\
\hline 0 & to & $4 e-7$ & & & & & & & & \\
\hline $4 e-7$ & to & 0.1 & $5.37(2) E+10$ & $67 \%$ & $1.44(2) E+10$ & $78 \%$ & $5.15(2) E-13$ & $14 \%$ & $2.30(2) E-13$ & $14 \%$ \\
\hline 0.1 & to & 1 & $4.18(2) E+09$ & $5 \%$ & $3.02(3) E+09$ & $16 \%$ & $1.14(2) E-12$ & $30 \%$ & $8.38(2) E-13$ & $48 \%$ \\
\hline \multirow[t]{2}{*}{1} & to & 20 & $1.59(5) E+09$ & $2 \%$ & $6.16(5) E+08$ & $3 \%$ & $1.96(5) E-12$ & $52 \%$ & $6.65(4) E-13$ & $38 \%$ \\
\hline & Tot: & & $8.07(2) E+10$ & $100 \%$ & $1.85(2) E+10$ & $100 \%$ & $3.76(3) E-12$ & $100 \%$ & $1.74(3) E-12$ & $100 \%$ \\
\hline
\end{tabular}

In order to perform calculations, a fine discretization in energy was implemented in the DPA cross-section, and results were obtained from the following formula:

$$
R_{\mathrm{DPA}} \cong \sum_{i=1}^{N} \bar{\sigma}_{D i} \int_{E_{i-1}}^{E_{i}} \Phi\left(E_{i}\right) d E_{i}=\sum_{i=1}^{N} \bar{\sigma}_{D i} \cdot \phi_{i}
$$

where $\sigma_{i}$ and $\phi_{i}$, are the microscopic cross-section and the flux in the $i$ group, and $N=89$ the number of groups.

Normalization was done with the number of fission neutron due to the power developed $(\approx 24 \mathrm{MW})$ by the fraction of FE (fuel) present in the truncated geometry and obtained from a calculation with the complete model normalized at $2160 \mathrm{MW}$.

4.2. Rate of DPA in the RPV Inner Surface. In Figure 8, level curves for constant DPA rate on the inner surface of the RPV are plotted using the values obtained with MCNP5.

A peak in the lower right corner of the graph $(Z=$ $33.75 \mathrm{~cm}, \theta=28.44^{\circ}$ ) may be observed and, due to the symmetries, an equivalent maximum should be present at the right hand of plane $\theta=30^{\circ}$ and, therefore, similar areas below plane $Z=0 \mathrm{~cm}$. The peaks that correspond to a mean value at the first centimeter of the RPV thickness is 3.76(3) $\times$ $10^{-12} \mathrm{~s}^{-1}$ where the percentage error of the determination is in brackets.

4.3. Rate of DPA in the Wall of RPV in the Area of the Maximum. In Figure 9, the level curves of the DPA rate on the wall of the RPV are plotted at the level corresponding to the maximum detected.

It may be noted that the level curves of constant DPA follow the contours of the "four" FE closest to the wall of the RPV. Thus, the estimated maximum rate of DPA, 3.76(3) $\times 10^{-12} \mathrm{~s}^{-1}$, will occur on the inner surface of RPV in front of BL43 and BA42 fuels of the A zone and LH03 and LG04 in the $\mathrm{B}$ zone.

4.4. DPA and Neutron Flux Spectrum. In Figure 10 the integral DPA rate in each energy group is plotted as a function of neutron energy in the zone of maximum value on the RPV cladding of $0.6 \mathrm{~cm}$ thickness, on the inner surface of the RPV, and at a depth corresponding to $1 / 4 \cdot T$, where $T$ is the thickness of the RPV. All curves are normalized to the maximum DPA rate $\left(4.31 \times 10^{-12} \mathrm{~s}^{-1}\right)$ obtained on the inner surface of the cladding. In Figure 10, we used linear interpolation instead of horizontal step for readability.
From Figure 10 we can see that as radiation penetrates the RPV, neutron spectrum hardens, reduces the rate of DPA and accordingly decreases the contribution of thermal neutron damage.

This statement can be translated into numbers observed in Table 1 which presents the spectrum of neutron flux and the rate of DPA for the four commonly used energy groups for two of the positions mentioned earlier.

From Table 1 it may be observed that the greatest contribution to DPA rate on the inner surface of the RPV in the maximum point corresponds to the fast flux (with 52\% of the total) and the first epithermal group (with 30\%). These contributions together correspond to a small fraction of the total flux $(7 \%)$ if the contribution due to the thermal flux (that is $26 \%$ of the total flux) is very small. However, for $R=$ $377.0 \mathrm{~cm}$ ( $\cong 1 / 4 \cdot T$ thickness), the greatest contribution to the DPA rate (with $62 \%$ of the total) is due to both epithermal groups corresponding to $94 \%$ of the total flux. The $38 \%$ of the remaining damage is due to the fast flux (that is $3 \%$ of the total flux) being the corresponding one to the thermal group negligible that for this position is $2 \%$ of the total flux.

It may be noticed that the maximum DPA rate in the first millimeter of the RPV cladding was also determined, giving a value of $4.31(3) \times 10^{-12} \mathrm{~s}^{-1}$.

\section{Comments and Conclusions}

As it was mentioned, the calculation was made using fresh FE. The estimation carried out is a minimum value because when the reactor begins to operate at equilibrium burnup, a neutron flux flattening occurs in the core center with the consequent growth in the periphery. It is this zone that contributes to neutron radiation damage. The fast neutron flux increases near the RPV wall thus producing an increase in the DPA rate.

Besides, it should also be taken into account that with increasing fuel burn-up, and as ${ }^{235} \mathrm{U}$ disappears and other fissile nuclides like plutonium appear, the $v$ value (number of neutron produced per fission), here used to normalize calculation, could increase $12 \%$, changing from approximately 2.45 to 2.75 from fresh to burned fuel (around $10.000 \mathrm{MWd} /$ tonU). This effect also contributes to increasing the DPA rate on the RPV wall.

The rate of DPA calculated by NA-SA (the utility) for the same region, an equilibrium burn-up core is 3.86(3) $\times 10^{-12} \mathrm{~s}^{-1}$, just a little bit greater than the estimation performed with this method. 
It may also be deduced that the presence of neutron poisons in heavy water such as $\mathrm{B}^{10}$ would not be an effective protection against neutron radiation damage to the RPV, since, as it can be seen from its cross-section in Figure 11, it only acts as a thermal poison not affecting either fast or epithermal neutron flux, the most important contributors to the DPA rate.

As a result of this work, a tool for the calculation of DPA rate in the RPV of Atucha II has been built at Argentine Regulatory Authority. For future work, a better and more realistic estimation of maximum rate of DPA could be done using the complete model with equilibrium burn-up core and including control rods at nominal position.

\section{Acknowledgment}

The authors are grateful to Dr. Ana M. Lerner for her useful comments about the present paper.

\section{References}

[1] G. S. Was, Fundamentals of Radiation Materials Science-Metals and Alloys, Springer, Berlin, Germany, 2007.

[2] "MCNP5-A General Monte Carlo N-Particle Transport Code, Version 5," Los Alamos National Laboratory, by the X-5 Monte Carlo Team, LA-UR-03-1987, April 2003.

[3] J. A. Mascitti, "Modelo tridimensional de Atucha II con MCNP5,” PE-IT-032, ARN.

[4] J. M. Longhino and H. R. Blaumann, "Modelado y Cálculo Neutrónico con MCNP de los parámetros relacionados al daño por radiación del Recipiente de Presión de Atucha II," Tech. Rep. CNEA-CAB 47/043/2006, Rev. 1, Comisión Nacional de Energía Atómica.

[5] http://www-nds.iaea.org/irdf2002/data/irdf2002_damage.dat. 

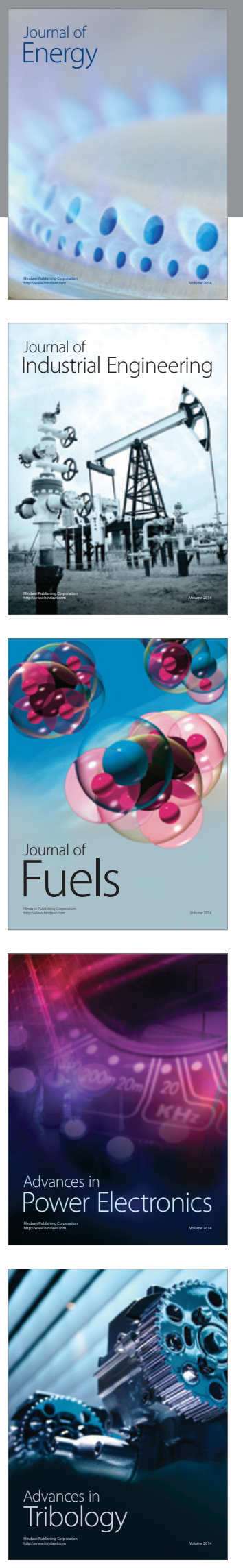
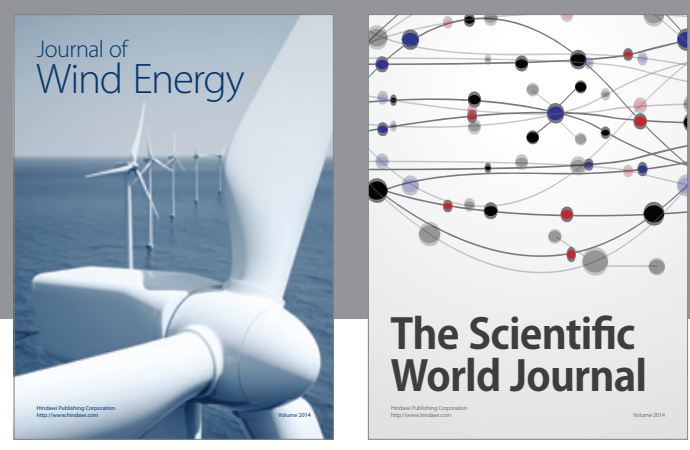

The Scientific World Journal

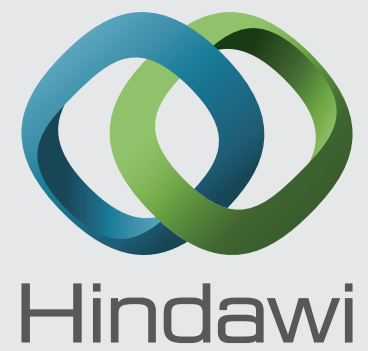

Submit your manuscripts at http://www.hindawi.com
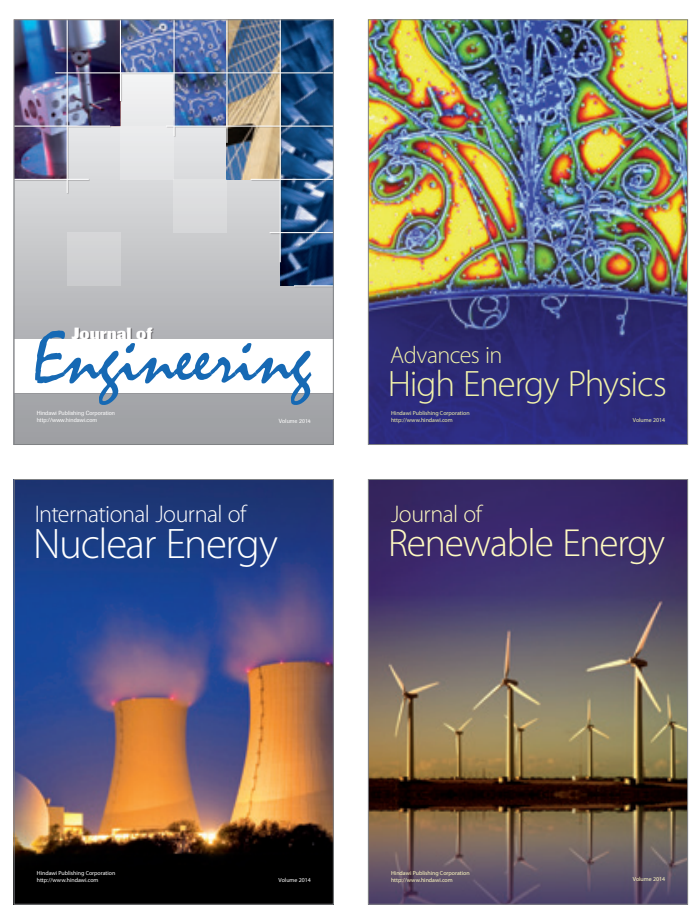

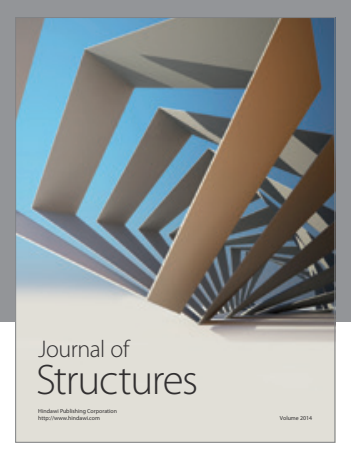

Rotating
Mechinery
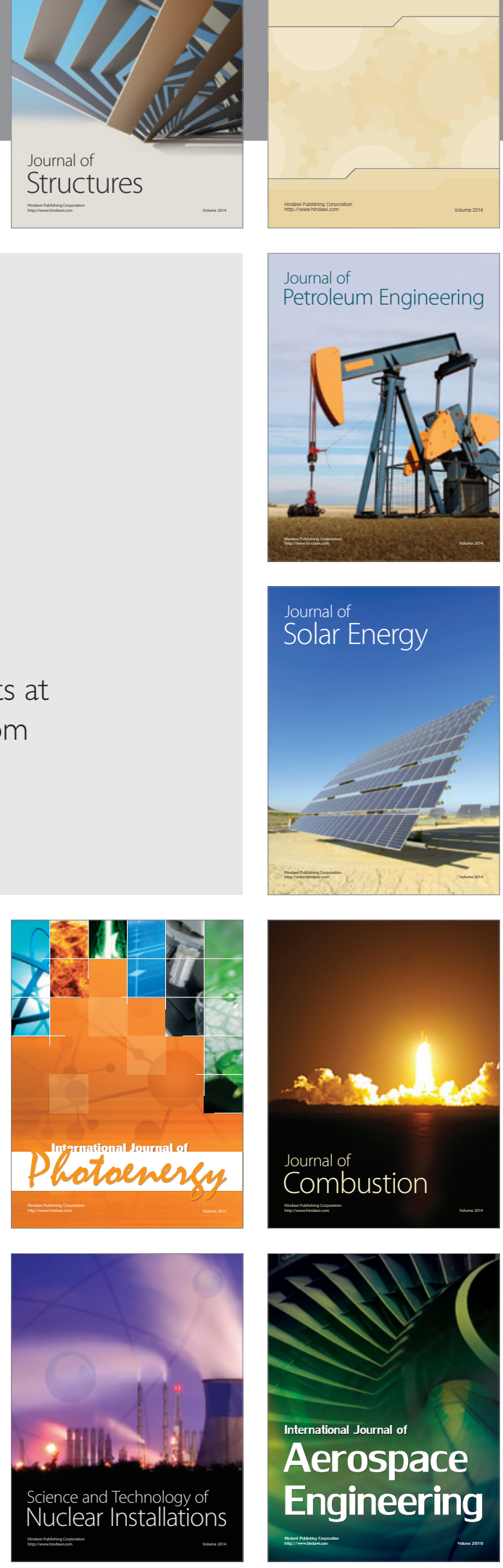